\title{
4-dimensional strain imaging of the right ventricle: application in patients with severe pulmonary hypertension
}

\author{
Alessandro Satriano ${ }^{1,2^{*}}$, Vijay Kandalam ${ }^{1,4}$, Khalil Jivraj $^{3}$, Yoko Mikami $^{1}$, Hanna Medwid ${ }^{5}$, Carmen Lydell ${ }^{1,3}$, \\ Naeem Merchant ${ }^{1,3}$, Andrew G Howarth ${ }^{1,2}$, Tracy L Elliot ${ }^{1,3}$, James A White ${ }^{1,2}$
}

From 18th Annual SCMR Scientific Sessions

Nice, France. 4-7 February 2015

\section{Background}

The risk stratification of patients with pulmonary hypertension (PHTN) using non-invasive techniques is challenging. 4D analysis techniques of right ventricular (RV) strain may provide unique opportunities for the identification of high-risk individuals. However, conventional strain metrics, developed for left ventricular geometry, pose limitations for RV geometry and its associated fibre orientations. In this study we examine a novel 4D strain analysis tool affording calculation of axis-independent Principal strains for the assessment of right ventricular mechanics. Patients with mild-moderate and severe PHTN were studied in comparison to a young healthy volunteer cohort.

\section{Methods}

Fifteen patients with PHTN, defined as a mean pulmonary arterial pressure (PAP) $>25 \mathrm{mmHg}$ at right heart catheterization, and 18 young healthy volunteers were studied. Severe PHTN was defined as a mean PAP $\geq 55 \mathrm{mmHg}$. A standardized imaging protocol was performed using a 3T MRI scanner. Cine images were blindly analyzed for LV and RV volumes using commercial software (cvi42, Circle Cardiovascular Inc., Calgary). 4D strain analysis was performed using prototype software, generating a 3D end-diastolic mesh model of the $\mathrm{RV}$ and then deforming this model within a 4D displacement field, the latter contributed to by short and long-axis cine image data (Figure 1). Peak Principal Strain Amplitude (PPSA) and Normalized Time to Peak (NTTP) strain were obtained for each model node and a novel metric calculated called the "Normalized Strain Attainment Rate" (NorStAR), where NorStAR = PPSA / NNTP (Figure 1). The latter composite metric was hypothesized to represent global RV electro-mechanical recruitment.

\section{Results}

The mean age of the PHTN cohort and healthy volunteers was $62.5 \pm 6.4 \mathrm{yrs}$ and $26.6 \pm 5.8 \mathrm{yrs}$ respectively. Among those with mild-moderate PHTN $(n=10)$ the mean PAP was $39.2 \pm 9.4(\mathrm{n}=10)$ versus $62.4 \pm 9.0 \mathrm{mmHg}$ among those with severe PHTN $(n=5)$. As shown in Figure 2 a significant difference in NorStAR was identified between severe PHTN and healthy controls $(-30 \pm 10$ vs $-47 \pm 9, \mathrm{p}=0.002)$, while no difference was seen for mild-moderate PHTN $(-44 \pm 10$ vs $47 \pm 9, \mathrm{P}=$ N.S.). The capacity of NorStAR to identify patients with invasive hemodynamics of severe PHTN was incrementally assessed using ROC analysis. This achieved an AUC of 0.85 for the identification of severe PHTN with a threshold of -38 providing optimal sensitivity (80\%) and specificity (60\%).

\section{Conclusions}

In this pilot study, we demonstrate the feasibility of $4 \mathrm{D}$ Strain analysis using Principal Strains for the characterization of RV mechanical deformation in PHTN. A novel marker, NorStAR, accurately identified patients with invasive hemodynamic evidence of severe versus nonsevere PHTN and may be of value for the risk stratification of this population. 
a)
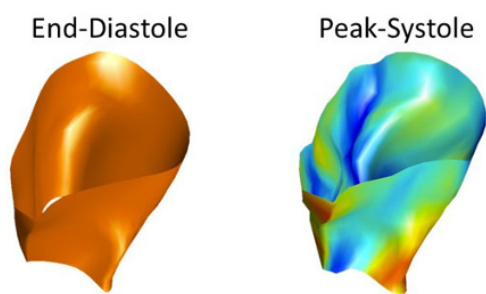

b)

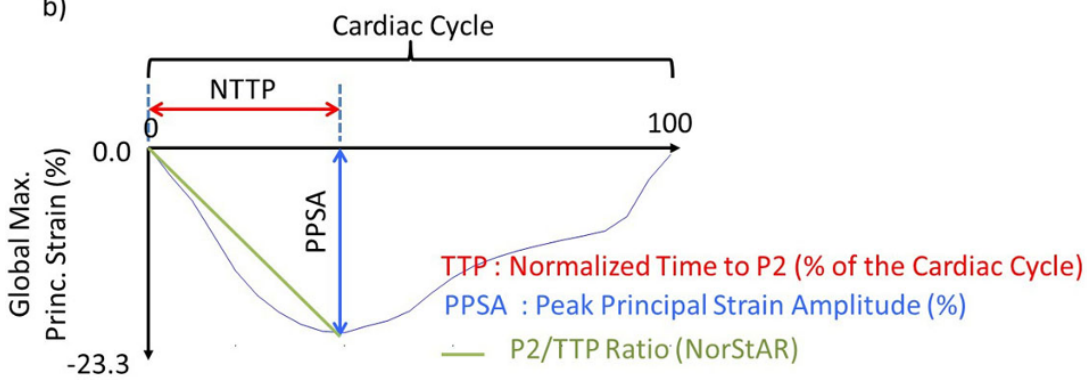

Max. Principal Strain (\%)

15.0

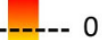

0 diastole and end-systole. (B) Illustration of the NorStAR calculation as the ratio between the Peak Principal Strain Amplitude (PPSA) and the Normalized Time To Peak (NTTP; as a percentage of the cardiac cycle).

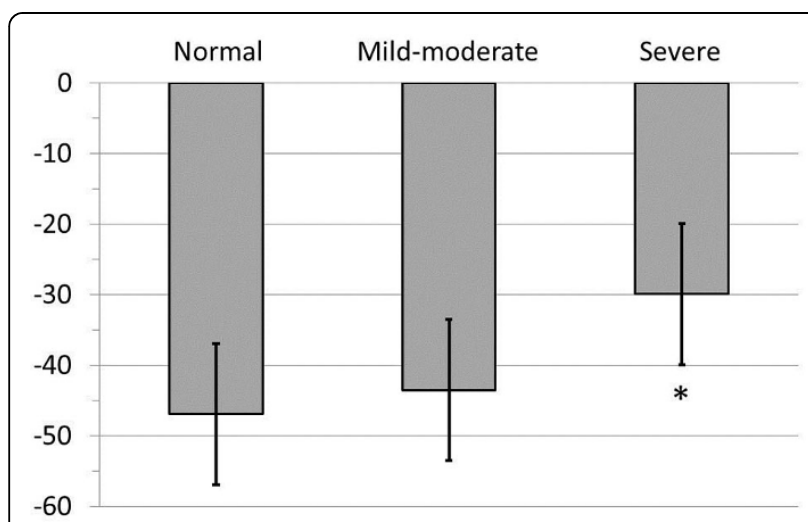

Figure 2 Mean values of the NorStAR strain parameter. NorStAR in healthy volunteers, mild-moderate patients, and patients with severe pulmonary hypertension, indicating a significant impairment in the severe group $\left({ }^{*} p<0.01\right)$.

\section{Funding}

This study was funded by the Calgary Health Trust. Dr Satriano receives support from Mitacs Canada and Medtronic of Canada, Ltd. Dr White is supported by a New Investigator Award from the Heart and Stroke Foundation of Alberta.

\section{Authors' details}

${ }^{1}$ Stephenson Cardiac Imaging Centre, Libin Cardiovascular Institute of Alberta, University of Calgary, Calgary, AB, Canada. ${ }^{2}$ Division of Cardiology, Department of Medicine, University of Calgary, Calgary, AB, Canada. ${ }^{3}$ Department of Diagnostic Imaging, University of Calgary, Calgary, $A B$, Canada. ${ }^{4}$ Department of Radiology, University of Calgary, Calgary, AB, Canada. ${ }^{5}$ Queen's University, Kingston, ON, Canada.
Published: 3 February 2015

doi:10.1186/1532-429X-17-S1-Q56

Cite this article as: Satriano et al:: 4-dimensional strain imaging of the right ventricle: application in patients with severe pulmonary

hypertension. Journal of Cardiovascular Magnetic Resonance 2015 17(Suppl 1):Q56.

\section{Submit your next manuscript to BioMed Central and take full advantage of:}

- Convenient online submission

- Thorough peer review

- No space constraints or color figure charges

- Immediate publication on acceptance

- Inclusion in PubMed, CAS, Scopus and Google Scholar

- Research which is freely available for redistribution 